\title{
Automated Discharge Planning Systems: Perceived Challenges and Recommendations
}

\section{Alghamdi $\mathrm{AA}^{1 *}$ and Taghreed $\mathrm{IJ}^{2}$}

${ }^{1}$ King Abdulaziz Medical City, Jeddah, Saudi Arabia

${ }^{2}$ King Saud bin Abdulaziz University for Health Sciences, Saudi Arabia

\begin{abstract}
Objective: This study aims to investigate the effectiveness of the current discharge planning program at the National Guard Health Affairs facility, King Abdulaziz Medical City-Jeddah, and to study the feasibility of introducing an automated discharge planning system in order to improve the discharge planning process.
\end{abstract}

Design: This study used a qualitative research design where a face to face interviewing technique was applied with the discharge planning team and patients to understand the benefits and barriers of the current discharge planning processes. A digital recording for the interviews was applied followed by transcribing these interviews by a professional transcriptionist and finally a comprehensive analysis was undertaken to understand the facts of the current discharge planning program in King Abdulaziz Medical City-Jeddah.

Results: The comprehensive analysis of the interviews showed that there is improvement in the discharge planning processes in comparison to previous years with lower-admission rate. It was also found that there are now several departments involved in the discharge planning program, whereas in the past there were only unorganized efforts by individual healthcare providers. It was also evident from the findings that there are significant factors that affect the discharge planning processes; (1) Severe shortage of discharge planning manpower; (2) Discharge planning service is undertaken by a group of healthcare providers working under different departments not under one management; (3) Some patients cannot be discharged due to social and financial reasons; (4) lack of an automated discharge planning system.

Conclusion: The current discharge planning processes at King Abdulaziz Medical City-Jeddah seems to be satisfactory. However, there are crucial factors that need to be rectified in order to optimize this program. Increasing manpower and developing an automated discharge planning system are recommended as significant elements to improve this service.

Keywords: Automated discharge; Hospitals; Patients; Patient leaves

\section{Introduction}

The traditional role of hospitals and medical centers that admit patients is to discharge those patients upon recovery without further medical or social care. More recently this role has shifted where hospitals and medical centers provide comprehensive medical and social care programs for the patient during his stay and after discharge [1]. These types of programs are referred to as Discharge Planning programs. The concept of discharge planning is to investigate the patient's medical, social, and financial needs that might affect his/her health after discharge to prevent or reduce re-admitting the treated patient [2]. The discharge planning processes start before the patient leaves the hospital where a professional team study the patient's needs including medications, education, medical equipment and even scheduling home visits to avoid the patient's re-admission [3]. Preventing or reducing patients' re-admission will benefit both the patients and the healthcare center [4]. Cost, bed utilization, and quality of care are the major factors that encourage healthcare centers to apply discharge planning [5]

\section{Importance of Discharge Planning}

Discharging a medical inpatient usually requires a multidisciplinary team and is a complex process [6]. It aims to improve the coordination of services after the patient is discharged by considering the patient's needs after leaving the hospital [2]. Its concept is to prevent or reduce the rate of patient readmission. Healthcare organizations aim to reduce or completely prevent hospital readmissions by implementing discharge planning [7]. Any patient who is admitted into the hospital after being discharged within 30 days is known as a readmission [8]. The benefits of reducing readmission rates include better bed utilization, medical treatment cost-effectiveness, and better patients' outcomes [9]. In a study conducted by Jack et al. [10], they found that when providing patients with a package of discharge services, hospital utilization was reduced thirty days after discharge. Unnecessary length of stays and over usage of hospital resources due to a lack of discharge planning can be avoided by implementing one [10]. Moreover, a study on older patients with congestive heart failure (CHF) who had a comprehensive discharge planning plus post discharge support showed that there was significant reduction in readmission rates and improvement in the patients' outcomes without increasing costs [11]. Another study by Benbassat and Taragin showed that $9 \%$ to $48 \%$ of all readmissions could be prevented since these cases received substandard care during the hospitalization such as poor treatment, improper therapy at discharge, and inadequate post-discharge care. Furthermore, randomized prospective trials have shown that $12 \%$ to $75 \%$ of all readmissions

*Corresponding author: Abdulmajid Abdullah Alghamdi, King Abdulaziz Medical City, Jeddah, Saudi Arabia, Tel: +966126240000; E-mail: GhamdiAA@ngha.med.sa

Received August 10, 2016; Accepted August 27, 2016; Published September 03, 2016

Citation: Alghamdi AA, Taghreed IJ (2016) Automated Discharge Planning Systems: Perceived Challenges and Recommendations. Health Care: Current Reviews 4: 173. doi: 10.4172/2375-4273.1000173

Copyright: (c) 2016 Alghamdi AA, et al. This is an open-access article distributed under the terms of the Creative Commons Attribution License, which permits unrestricted use, distribution, and reproduction in any medium, provided the original author and source are credited. 
can be prevented by patient education, pre-discharge assessment, and domiciliary aftercare [12]. In light of these benefits, many countries have started to implement discharge planning programs within their healthcare systems as a means to improve patient outcomes and to reduce readmission rates [13].

\section{Manual to Automated Discharge Planning}

A discharge planning system has the potential to make the patients' discharge process a smooth transition, thereby limiting the waste of hospital time and funds [14]. A new field of interest in discharge planning is the movement from manual to computerized systems. While the implementation of a discharge planning system in itself has the potential to ease patients' discharge, it can still be deficient in many aspects [15]. Planning the discharge of a patient from a hospital can be lengthy and time consuming [16]. An assigned discharge planning coordinator must start the discharge process from the day of admission [17]. This process consumes a significant amount of paper work to ensure that the discharged patient has received proper care and is safe to leave the hospital [18]. Multidisciplinary team of medical and nursing professional healthcare providers is involved in the process of discharge planning and all of them communicate with each other by using traditional old communication tools such as fax machines which delay the discharge process and may reduce the quality of care [19]. Some of the disadvantages are poor communication and coordination between the planning teams resulting in delayed discharge and unnecessary extensions of length of stay for the patients [20]. As a result of increased length of stay, hospital overcrowding occurs that in turn increases the bed shortage crisis that most hospitals suffer from [21]. According to previous studies, nurses complained that the biggest barrier to successful discharge planning was lack of time due to high workload, poor sharing of information between teams and unplanned discharges $[22,23]$. To help resolves these issues an electronic discharge system can be implemented to overcome the obstacles of miscommunication, delayed information relay between teams and helps to better advance discharge planning for a smoother process.

\section{Mobilizing an Electronic Discharge Planning System}

An electronic discharge system could be an online, real time program that can be utilized from desktops, laptops, personal digital assistants (PDA), tablets or smart phones [19]. By widening the access to the system, delay in sending the important discharge information about the patient will be minimized. Electronic handheld devices have solved the issue of needing to access a system from a specific place. The advantages of applying a system to hand held devices such as iPads and smartphones can increase information access, promote workflow and allow the physicians to make decisions from miles away with the ability to access the patient's data, keeping in mind the need for a safe, secure network to protect patients confidentiality [24].

\section{Electronic Discharge Planning in Real Time}

A study was conducted at Johns Hopkins pediatric intensive care unit to develop real-time length of stay prediction and patient flow management system [25]. The model software used in the study was designed to be integrated within a computerized decision support system to improve patient flow management [25]. The result showed that the provider orders are useful for real-time prediction of patients' length of stay. Clinical orders directly represent provider decision making over time. The provider orders for real-time analysis make all information available in real-time through single computerized physician order entry (CPOE) data [25]. Such systems provide accurate and timely length of stay forecast which could be a very useful tool for healthcare organizations management and planning [25]. Such model software's using real-time systems would be like an application that generates a user interface allowing each team to enter their plan for a patient on the same server. All working simultaneously to make sure the patient discharge information is accurate and complete. Some of the shared information would the patient's name, MRN, diagnoses, current medications and allergies to name a few [19]. Other aspects of such a system would be a way to keep track of the patients progress since admission including all investigations and procedures done, including a way of know where the patient is at all time so as no overlapping between schedules procedures occurs and prioritizing them according to critical importance [26].

\section{Difficulties of an Electronic Discharge System}

Ideally a custom made computerized discharge planning system would bring about solutions to the manual discharge process, many pitfalls may occur while trying to implement such a system. The main difference would be the staff inability to interface with the complex system. Staff would need to train in how to correctly utilize the software which would increase workload and give added responsibilities [23] Another important issue is data security. The data on this kind of system would be extremely sensitive and patient privacy is a top issue [24]. The ramifications of a leak of any kind would have extreme repercussions including lawsuits and liabilities.

\section{Example of Discharge Planning System Software}

Multiple software companies have created electronic discharge planning programs that are tailored to the needs of a healthcare organization. Such companies include Patient Placement, which created 'The Patient Placement Discharge Referral System' that speeds up the process of patient admission and discharge [27]. The benefits of incorporating a system such as this would make a profound effort in simplifying and hastening the discharge process.

\section{Importance of Automated Discharge Planning}

With all aspects considered, an automated discharge planning system is highly advantageous and promotes a better quality of care for patients. Through a successful discharge plan including all departments with a complete treatment plan and patient education, a patient's quality of life and satisfaction is high after discharge from the hospital [28]. Smooth transition for the discharged patient from the hospital to home or to the referred primary care physician can be achieved by automating the discharge planning processes [29]. This study aims to emphasize the needs for such systems.

\section{Discharge Planning in KAMC - Jeddah}

Discharge planning is not a new concept in Saudi Arabia. King Abdulaziz Medical City - Jeddah (KAMC-Jeddah) is an example of a facility that has implemented discharge planning services for several years now. It consists of multidisciplinary health providers, which include; discharge planning specialists, physicians, nurses, pharmacists, physiotherapists, home health care specialists, etc. [30]. The discharge planning services' role is to coordinate with all involved healthcare providers to provide the discharged patients with all necessary medications, appointments, medical supplies, clinical and non-clinical instructions, and all necessary information that the patient may need to prevent or minimize readmission events. However, the discharge planning team is still not capable of providing adequate services to the discharged patients due to the lack of proper coordination and 
communication between the concern healthcare providers [31]. This paper presents a qualitative study that conducted at KAMC-Jeddah, to examine these issues by interviewing the discharge planning team, the discharged patients and discharged patients' families.

This study aims to investigate the effectiveness of the discharge planning program within the National Guard Health Affairs facility, KAMC-Jeddah, and the feasibility, and usefulness of introducing an automated discharge planning system. During the course of the study, more specific objectives are also addressed, such as recognizing the benefits and outcomes of discharge planning; identifying the appropriate discharge planning members; determining the appropriate discharge planning process; analyzing the feedback from the discharge planning patients; determining the barriers that have hindered introducing an automated discharge planning system so far; and understanding the perceived advantages to introducing an automated discharge planning system.

\section{Materials and Methods}

\section{The participants}

The individuals interviewed for this study were selected from the different departments, particularly the healthcare professionals who are involved in the discharge planning processes. The interviewees were; Director of Discharge Planning, Medical Discharge Planning Coordinator, Surgery Discharge Planning Coordinator, Cardiac Center Discharge Planning Coordinator, Assistant Director for Admission, Assistant of Inpatient Care Director, Social Worker, and Patient Educator. Also, five patients and five patients' relatives were interviewed. The above group was selected as they were involved directly in the discharge planning process on a daily basis. The patients and their families were selected for this interview because they are the receivers of the service and their thoughts and satisfaction are valuable for this study. The total number of face to face interviews for this study was eighteen.

\section{Study design and method}

A qualitative method was applied for this research since more understanding of people's feelings, thoughts, motivations, and reactions is involved here rather than numbers, which is usually the point of applying quantitative methods. Also, this research targeted a small and focused group of people to explore specific views and to have rich and in-depth understanding of a specific situation which cannot be achieved by a quantitative method that tends to give general views and results generated by a big scale of population. There was human involvement in this research where face to face interviews were conducted in order to explore unexpected results that cannot be achieved by quantitative methods [32].

\section{Sampling}

A nonprobability sampling technique was applied, and two types of sampling were used to interview the healthcare givers who are involved in the discharge planning processes:

\section{Snowball Sampling}

2. Purposive Sampling

Both sampling techniques were used until theoretical saturation was achieved [32].

\section{Structured qualitative interviews}

A close-ended structured survey containing dichotomous questions was applied to interview the patients and the patients' families. These were administered face-to-face. Participants were asked straightforward questions and their responses were recorded directly. All participants were asked to answer the same questions as is the nature of closed-ended interviews (Appendix 1).

\section{Semi-structured qualitative interviews}

Open-ended semi-structured interviews were conducted with the healthcare providers. The format of the interview was guided by an interview guide (Appendix 2). However the participants were allowed to elaborate more freely when answering the questions. The healthcare providers' interview questions were divided to three sections; (1) Discharge Planning Members; (2) Discharge Planning Processes; (3) Automating discharge planning Process. These questions were designed to achieve maximum understanding of the current discharge planning processes and the possibility of introducing an automated discharge planning system.

\section{Research Analysis}

A digital audio recorder was used to record the interviews with the health care providers, followed by transcribing all audio recordings by a professional transcriptionist. For reliability a backup recorder was also used so that no information was lost.

A qualitative Content Analysis approach was applied to analyze the text data [32]. The data was resolved to five predetermined themes; (1) Benefits; (2) Discharge Planning Team; (3) Discharge Planning Processes; (4) Barriers for Discharge Planning; (5) Applying Automated System.

The benefits theme discussed the gain of the discharge planning processes in KAMC - Jeddah for both the patient and the healthcare facility. The Discharge Planning Team theme investigated the healthcare providers who have direct contacts with the patients during the discharge planning processes. The investigation discussed the availability of the discharge planning team, their roles, competency, and authority. The Discharge Planning Processes theme discussed how the discharge planning has been conducted in KAMC - Jeddah and the related problems of the discharge planning processes. The Barriers for Discharge Planning theme discussed the problems that affected the processes of the discharge planning including manpower, uncooperative patients and their families, lack of communication between discharge planning team, and the absence of automated discharge planning system. Finally, the Applying Automated System theme discussed how the current discharge planning processes have been conducted manually, and the feasibility of applying an automated system to help in improving the discharge planning processes (Table 1).

\section{Discussion}

\section{Benefits of discharge planning}

"Patient don't come back, especially long stay patient, when provided with HHC they never come back" (Surgical Discharge Planning Coordinator). The discharge planning processes helped in reducing the re-admission rate especially when Home Health Care (HHC) services is provided for the long stay patients who need only a nursing care (Surgical Discharge Planning Coordinator). Also, the re-admission rates for Oncology patients who are occupying a big percentage of bed resources are now reduced due to the discharge planning (Cardiac Center Discharge Planning Coordinator). The average rate of length of stay was also reduced compare to several years 


\begin{tabular}{|c|c|}
\hline Themes & Findings \\
\hline Benefits & $\begin{array}{l}\text { - Reduce re-admission rate } \\
\text { - Reduce the length of stay } \\
\text { - Physicians are also benefit from the system }\end{array}$ \\
\hline $\begin{array}{l}\text { Discharge } \\
\text { Planning } \\
\text { Team }\end{array}$ & $\begin{array}{l}\text { - Severe shortage in discharge planning staffing } \\
\text { - Lack of expert and skillful manpower. } \\
\text { - Need to develop a department to empower the discharge planning } \\
\text { services }\end{array}$ \\
\hline $\begin{array}{l}\text { Discharge } \\
\text { Planning } \\
\text { Processes }\end{array}$ & $\begin{array}{l}\text { - Hand-over every morning } \\
\text { - Discuss every detail and problem before discharging the patient } \\
\text { - Daily meeting with the Discharge Planning Director to discuss } \\
\text { every patient issues } \\
\text { - All clinical tests and social studies are completed before } \\
\text { discharge } \\
\text { - All medical equipment, medical supplies and sick leave certificate } \\
\text { are provided to the patient before discharge } \\
\text { - The Discharge Planning processes start upon the admission of } \\
\text { the patient } \\
\text { - Still, the current practice is not efficient and should be revised } \\
\text { - Discharge planning processes should be modified in } \\
\text { - Conflicts in physicians decisions delay discharge } \\
\text { - Hospital rounds are not done on time, which delay discharge } \\
\text { planning processes } \\
\text { - Very late call for patient assessment } \\
\text { - JCl standards and App for Patient Care are now applied } \\
\text { Weekly meeting for discharge planning team. }\end{array}$ \\
\hline $\begin{array}{l}\text { Barriers for } \\
\text { Discharge } \\
\text { Planning }\end{array}$ & $\begin{array}{l}\text { - No discharge planning department or inpatient care department, } \\
\text { only services coordinated by multiple departments } \\
\text { - Severe shortage in discharge planning staff } \\
\text { - Lack of authority for discharge planning team } \\
\text { - Pate of the discharge day is not planned upon admission } \\
\text { - Patients having non-medical reasons which delay discharging } \\
\text { verbally and not documented } \\
\text { - Poor communication between discharge planning team members } \\
\text { - Family refusal for discharge due to patient care burden at home } \\
\text { - Patient with a tracheostomy cannot be discharged } \\
\text { - Long stay patients staying for indefinite period } \\
\text { - Physicians do not see patients upon admission } \\
\text { - Unreported patients for discharge planning team } \\
\text { - Last minute call to educate the patient and the family } \\
\text { - Patients referral documents are written manually and they go } \\
\text { - Patient discharge plan are not requested on time } \\
\text { - Anonymous and neglected patients } \\
\text { - Uncooperative patients } \\
\text { - Bed utilization is not according to the international standards }\end{array}$ \\
\hline $\begin{array}{l}\text { Applying } \\
\text { Automated } \\
\text { System }\end{array}$ & $\begin{array}{l}\text { - Currently, all patients' documents are processed manually } \\
\text { - Communication between discharge planning team members are } \\
\text { done through telephone and pagers, not by computerized system } \\
\text { - Automated discharge planning system is required to help } \\
\text { controlling the discharge planning processes } \\
\text { - A discharge planning automated system is existing in King } \\
\text { Abdulaziz University Hospital } \\
\text { - The Automated discharge planning system should include } \\
\text { Computerized Physician Entry Order (CPOE) } \\
\text { - Automated discharge planning system will also be beneficial for } \\
\text { Admission Processes } \\
\text { - The system will track the progress of the patient instantly for all } \\
\text { discharge planning team members. } \\
\text { - An automated discharge planning system has been developed } \\
\text { but not completed yet }\end{array}$ \\
\hline
\end{tabular}

Table 1: Automated discharge planning systems.

ago (Social Worker). Not only patients benefit from the discharge planning, but also physicians benefit from the discharge planning process as it alerts them about the patients need (Medical Discharge Planning Coordinator)

\section{Discharge Planning Team}

"We need more manpower, and this is not only the number, we are looking for quality of manpower" (Director of Discharge Planning)
Multiple departments undertake the discharge planning process in KAMC - Jeddah. However, there is a severe shortage in the discharge planning team who can cover the whole medical city (Medical Discharge Planning Coordinator). There are only 2 discharge planning nurses who should deal with over 600 in-patients (Assistant of Inpatient Care Director). During bed crises Situations, the efficiency of the discharge planning is only $20 \%$ to $30 \%$, due to insufficient number of discharge planning manpower (Assistant Director for Admission). For example, the Home Health Care Department which has a big role in discharge planning should be provided with more manpower to increase its efficiency (Director of Discharge Planning). The discharge planning team members are not only insufficient, but also need extra manpower with high quality in this field (Director of Discharge Planning). Each team needs a sub-specialty coordinator to overcome the discharge planning obstacles (Medical Discharge Planning Coordinator). One of the major disadvantages of discharge planning is there is not a discharge planning department to empower the services and control the processes (Assistant Director for Admission).

\section{Discharge Planning Processes}

"We make sure that the patient goes home with all needed equipment and the social support" (Cardiac Center Discharge Planning Coordinator) The Discharge Planning processes start immediately upon the admission of the newly admitted patient (Cardiac Center Discharge Planning Coordinator). During the hand-over in every morning between the night shift staff and the morning shift staff, the discharge planning coordinators review and discuss the progress of each patient. Also, the discharge planning team has a daily meeting with the Discharge Planning Director to discuss the medical and social condition of admitted patients (Surgical Discharge Planning Coordinator). In addition, a weekly, meeting is conducted to discuss discharge planning issues including all services that are involved in the discharge planning such as Home Health Care, Pharmacy, Social Work Services, Occupational Therapy, Nursing Services, and Admission office (Medical Discharge Planning Coordinator). Before discharging the patient, all clinical tests, social studies and patient education are completed. Also, all medical equipment, medical supplies, outpatient appointments, and sick leave certificate are provided to the patient before discharge (Cardiac Center Discharge Planning Coordinator). The discharge planning process in KAMC - Jeddah are now complying with the Joint Commission International (JCI) standards (Social Worker). However, the current implementation of the discharge planning procedures is not efficient for several reasons and should be revised (Cardiac Center Discharge Planning Coordinator). For instance, the different views and decisions of the treatment responsible physicians cause confusion for both the patient and the discharge planning team which delays the discharge planning processes (Director of Discharge Planning). Another problem that affects the discharge planning process is the daily hospital round by the practitioners and healthcare givers (Assistant of Inpatient Care Director). The patient Educators are called to assess and educate the patients how to use medications and medical devices at home (Patient Educator).

\section{Barriers for Discharge Planning}

"You cannot cope with the number of patients we have and the number of beds available. This leads sometimes that patients prematurely discharged to evacuate beds, and that caused readmission" (Assistant of Inpatient Care Director).

KAMC - Jeddah provides discharge planning services, and there are internal guidelines to be applied by all departments involved 
in discharge planning. However, there is not a discharge planning department with its own manpower and budget, and its services are provided by a small team consists of seven staff only headed by the Discharge Planning Director, who is also the Inpatient Care Director. This small team must follow the needs of over six hundreds patients in a daily basis, coordinates with other departments, and assure that each patient is served with high quality of healthcare. This inappropriate setup does not give the discharge planning team the authority to follow up discharge planning issues with other departments (Assistant of Inpatient Care Director). The discharge planning services depends on cooperation and harmony between the service provider from all concerns departments rather than planned work with firm system. The patient orders and needs are communicated between those providers verbally, and there are not written orders to be used as medical or administrative references (Surgical Discharge Planning Coordinator). The communication between the discharge planning team members is poor due to the absence of an organized department and a computerized system that all discharge planning workers refer to in order to see patient progress and medical, nursing and social needs (Social Worker). It is very crucial that the responsible physician should see the admitted patient once he/she is admitted to develop the treatment plan, which is the baseline for the discharge planning. However, physicians are not compliance with this procedure and patients could be lying in bed for several days without a clear treatment plan or estimated discharge day, which affect the whole discharge planning process (Assistant of Inpatient Care Director). Occasionally, patients are admitted without notifying the discharge planning team, which delay the service and affect the patient care. Also, there are cases where Anonymous patients are admitted without a pervious medical history or family contacts. Those patients are difficult to prepare a discharge plan for them due to the absence of family involvement in their treatment (Social Worker). Last minute call to educate the patient and the family is another problem that delays the discharge planning process. Particular diseases need long session to educated patients and their families how to use medication or medical devices at home. Calling the Patient Educators right before discharge for educational session will not help the patient and may cause re-admission for the patient (Patient Educator). In general patients and their families are cooperative but there are cases where both are not welling do comply with the treatment plan and therefore they become regular visitors to the admission wards. Those patients make the discharge planning processes useless and meaningless (Medical Discharge Planning Coordinator)

Long stay patients are the most difficult cases the discharge planning team members have to deal with. They are not capable to take care of themselves and their families refuse to accept the discharge since those disabled patients put a huge burden with their special nursing care. Patients with a tracheostomy cannot be discharged anyway due to their medical conditions, which make their staying in the hospital indefinite (Cardiac Center Discharge Planning Coordinator).

Some patients are admitted for non-medical reasons, mostly social reasons, which need a careful assessment for their discharge planning. Routine discharge planning procedures are not applicable in these cases which make it difficult to develop discharge plan for them (Surgical Discharge Planning Coordinator). Unplanned admission is another problem that affects the discharge planning processes, especially the cases admitted through Emergency Room. This causes disruption for discharge planning team and admission office. Finally, the bed utilization is not according to the international standards. There is an estimated length of stay for each medical case, which is applied internationally. However, these standards are not followed, which makes discharge planning less affective (Assistant Director for Admission).

\section{Applying Automated System}

"If you can see the status and the progress of the patient, you can track the plan when patient will be discharged, and this will give you more effective way to utilize your bed, and make your planning" (Assistant Director for Admission) Currently, the discharge planning processes are distributed manually in KAMC - Jeddah. There is no automated system that can display the inpatient progress or orders for the concerned individuals and services that are involved in the discharge planning processes. Patient's referral documents are written manually and occasionally these documents cannot be located which delay the discharge planning processes (Surgical Discharge Planning Coordinator). Even Communication between discharge planning team members are done through telephone conversions and paging system to explain the need of patients, and not all the time the required member is available which affect the discharge planning process (Cardiac Center Discharge Planning Coordinator). An automated discharge planning system is required to coordinate and control the discharge planning processes. In this system all Computerized Physician Entry Order (CPOE), nursing orders, patient educators requests, social workers requests, and other orders and requests can be monitored and processed by all concerned providers immediately (Director of Discharge Planning). Furthermore, all discharge planning team members can monitor the progress of each patient and that would significantly help in discharge planning and bed utilization (Assistant Director for Admission). Although it is not widely existed in Saudi Arabia, but there are a few healthcare facilities already applied an automated discharge planning system. King Abdulaziz University Hospital in Jeddah has developed this system and it is helping its discharge planning processes. Implementing an automated discharge planning system in KAMC-Jeddah would certainly improve the discharge planning processes and reduce re-admission rate and that is the main goal of discharge planning (Director of Discharge Planning).

\section{Research Limitations}

This research had some limitations. A few of individuals who are involved in the discharge planning processes were not available for interviewing. Language barrier was another limitation since some of the interviewees preferred not to speak in English during the interview. Furthermore, since English language was not the native language for all the professional healthcare providers interviewees, the interviews and later transcribing and interpretation of the concept and idea of these interviews was difficult and time consuming, as can be seen in some of the interview excerpts: "Let say, patient needs MRI or CT, sometimes his schedule for 3 to 4 days with no salt, they keep the patient waiting. This is the problem in the operation of MRI or CT, we can see from that side, there is also no space for the team, they try their best to help, because the priority for the in-patent, to wait for the bed when the patient is discharged" (Interviewee 1).

This an example of an unstructured and confusing sentence said by an interviewee during the interview, that needed to be analyzed carefully after the transcription was completed to extract meaningful information. Another limitation was the unavailability of data for discharge planning patients or documents due to the lack of an automated discharge planning system. There was not an existing automated discharge planning system in other National Guard Health 
Affairs facilities to conduct manual processes vs. automated processes comparison for discharge planning.

\section{Ethical Considerations}

Interview consents from all interviewees were obtained including their approval to use any valuable information needed for this research. The interviewees were informed that their names will not be revealed and the information they provide will be used only for this research (Appendix 3). The purpose of this research was explained to each interviewee prior conducting the interview. The professional transcriptionist signed a confidentiality agreement not to disclose any information regarding the identities of the interviewee or the contents of the interviews (Appendix 4).

\section{Validity}

To validate this research, two researchers examined the content of the interviews transcriptions, results and analyses.

\section{Reliability}

The Reliability of this research includes:

1. Observing Discharge Planning Policy and Procedures (Appendix 5)

\section{The Quality of Digital Audio Recording}

3. Backup Recordings

4. Professional transcription

\section{Conclusion}

This research aimed to investigate the effectiveness of the current discharge planning processes in the KAMC - Jeddah. It also discussed the feasibility of developing an automated discharge planning system to improve the discharge planning program. Several healthcare providers from deferent departments who form the discharge planning team were interviewed to explore the current processes, benefits, barriers, and the feasibility of introducing an automated discharge planning system. Patients and patients' families were also interviewed to evaluate their satisfaction of the provided services. The study showed that there is a significant improvement in the quality of care. It also showed an improvement in the clinical outcomes for the discharged patients in comparison to the previous discharge processes when there was no organized discharge planning program. The study also proved that there is a significant effort from different departments to optimize the discharge planning processes in order to minimize the re-admission events for the discharge patients. However, this study showed that despite the noticeable effort from the discharge planning team, the current discharge planning processes requires more manpower involvement and introducing an electronic tool or automated system to improve the discharge planning processes. This research did not only discuss the advantages, disadvantages, and barriers to the existing discharge planning processes, but it also demonstrated the need for developing an automated discharge planning system. The major finding of this study is that computerizing the discharge planning processes is now essential as it is perceived to improve the communication between the discharge planning team, would shorten the response time to patient's treatment orders, and has the potential to provide instant report of the patient current status that can be viewed by all discharge planning team at any given time. Based on the participants' feedback. An efficient discharge planning processes enhanced by a computerized system would result in reducing the re-admission rate due to the planned clinical, social, and administrative care provided to the patient starting from the day of admission until discharge day. The study also revealed important information and facts that contradict the current assumption about discharge planning processes. Despite the current satisfaction by both the healthcare providers and the discharged patients, and the noticeable improvement in the discharge planning program in the recent years when compared to three years ago, there are many serious disadvantages that should be corrected to optimize this service. The current rate of satisfaction should not be considered as an evidence of high quality of discharge planning service. Increasing the number of manpower and introducing an automated discharge planning system are the main steps towards improving the discharge planning program in KAMC - Jeddah.

\section{Recommendations}

Introducing an automated discharge planning system at KAMCJeddah is essential to improve the discharge planning processes. This automated system could be developed either internally by the Information Services Department (ISD), or it could be acquired externally by a third party. Such system would help the discharge planning team to receive instant notification regarding the planned discharge patients through e-mail or text messages and respond immediately to prompt the discharge planning processes. The development of an automated discharge planning system has to be reviewed and undertaken by professional information systems developers.

\section{Summary}

\section{What was already known}

- Discharge planning service in KAMC-Jeddah does not have enough healthcare providers to apply adequate discharge planning for every discharged patient.

- There is not an automated discharge planning system that can help the discharge planning team to provide proper service.

\section{What this study added to our knowledge}

- The discharge planning team members in KAMC-Jeddah are very dedicated and willing to expand their services.

- There is not a universal automated discharge planning system that could be acquired and installed immediately. Each healthcare organization needs to tailor their own system according to their needs.

\section{Acknowledgement}

I would like to thank the Institutional Review Board (IRB), King Abdullah International Medical Research Center (KAIMRC) for reviewing my proposal and approving the conduct of this study. I also would like to thank the Research Funding Committee, KAIMRC to fund the expenses of this study. I would like to express my gratitude to everyone who supported me throughout the Master of Science in Health Informatics graduation project. Special thanks to the healthcare providers, patients and patients' families who shared with me their experiences and knowledge regarding my research. Their contribution was highly valuable to this study. I also would like to thank the professional Transcriptionist Ms. Famorcan whose professional transcription helped me in organizing my thoughts and the outputs of the study. Finally, My warmest thanks to Dr. Taghreed Justinia for her support and guidance during my research and during writing this project. Dr. Justinia' professionalism, ethics, and knowledge were the driving force for me to complete this study in a professional content.

\section{Conflict of Interest}

There was no conflict of interest in this study. 
Citation: Alghamdi AA, Taghreed IJ (2016) Automated Discharge Planning Systems: Perceived Challenges and Recommendations. Health Care: Current Reviews 4: 173. doi: 10.4172/2375-4273.1000173

\section{References}

1. Preen DB, Bailey BES, Wright A, Kendall P, Phillips M, et al. (2005) Effects of a multidisciplinary, post-discharge continuance of care intervention on quality of life, discharge satisfaction and hospital length of stay: A randomized controlled trial. Int J Qual Health Car 17: 43-51.

2. Katikireddi SV, Cloud GC (2008) Planning a patient's discharge from hospital.

3. Naylor M, Brooten D, Jones R, Lavizzo-Mourey R, Mezey M, et al. (1994) Comprehensive discharge planning for the hospitalized elderly: A randomized clinical trial. Annals of Internal Medicine 120: 999-1006.

4. Mudge AM, Shakhovskoy R, Karrasch A (2013) Quality of transitions in older medical patients with frequent readmissions: Opportunities for improvement. European Journal of Internal Medicine 24: 779-83.

5. Jackson MF (1994) Discharge planning: Issues and challenges for gerontological nursing. A critique of the literature. Journal of Advanced Nursing 19: 492-502.

6. Durstenfeld MS, Saybolt MD, Praestgaard A, Kimmel SE (2014) Abstract 353 Physicians do not accurately predict length of stay of patients admitted with heart failure. Circulation: Cardiovascular Quality and Outcomes 7: A353.

7. Hunter T, Birmingham J (2013) Preventing readmissions through comprehensive discharge planning. Professional Case Management 18: 56-63.

8. Jha AK (2014) Reducing readmissions: What might it take? Journal of the American Heart Association. J Am Heart Assoc 3.

9. Evans RL, Hendricks RD (1993) Evaluating hospital discharge planning: A randomized clinical trial. Medical Care 31: 358-370.

10. Jack BW, Chetty VK, Anthony D, Greenwald JL, Sanchez GM, et al. (2009) A reengineered hospital discharge program to decrease rehospitalizationa randomized trial. Annals of Internal Medicine 150: 178-187.

11. Phillips CO, Wright SM, Kern DE, Singa RM, Shepperd S, et al. (2004) Comprehensive discharge planning with postdischarge support for older patients with congestive heart failure: A meta-analysis. JAMA 291: 1358-1367.

12. Benbassat J, Taragin M (2000) Hospital readmissions as a measure of quality of health care: Advantages and limitations. Archives of Internal Medicine 160: 1074-1081.

13. Shepperd S, Lannin NA, Clemson LM, McCluskey A, Cameron ID, et al. (2010) Discharge planning from hospital to home. Cochrane Database Syst Rev 1.

14. Manfredi SM (2013) The design, implementation and evaluation of a technology solution to improve discharge planning communication in a complex patient population.

15. Wong E, Yam C, Cheung A, Leung M, Chan F, et al. (2011) Barriers to effective discharge planning: A qualitative study investigating the perspectives of frontline healthcare professionals. BMC Health Services Research 11: 242.

16. Chaboyer W, Foster M, Kendall E, James H (2004) The impact of a liaison nurse on ICU nurses' perceptions of discharge planning. Australian Critical Care: Official Journal of the Confederation of Australian Critical Care Nurses 17: 25-32.
17. Ortiga B, Salazar A, Jovell A, Escarrabill J, Marca G, et al. (2012) Standardizing admission and discharge processes to improve patient flow: A cross sectional study. BMC Health Services Research 12: 180.

18. Chaboyer W, Foster M, Kendall E, James H (2002) ICU nurses' perception of discharge planning: A preliminary study. Intensive and Critical Care Nursing 18: $90-95$.

19. Wyman RK (2011) Computer systems for managing patient discharge. Google Patents.

20. Waring J, Marshall F, Bishop S, Sahota O, Walker M, et al. (2014) Health services and delivery research. An ethnographic study of knowledge sharing across the boundaries between care processes, services and organisations: The contributions to 'safe' hospital discharge. Southampton (UK): NIHR Journals Library.

21. Simmons FM (2005) CEU: Hospital overcrowding: An opportunity for case managers. The Case Manager 16: 52-54.

22. Watts R, Pierson J, Gardner H (2006) Critical care nurses' beliefs about the discharge planning process: A questionnaire survey. International Journal of Nursing Studies 43: 269-79.

23. Watts R, Gardner H, Pierson J (2005) Factors that enhance or impede critical care nurses' discharge planning practices. Intensive and Critical Care Nursing 21: $302-313$

24. Lu Y-C, Xiao Y, Sears A, Jacko JA (2005) A review and a framework of handheld computer adoption in healthcare. International Journal of Medical Informatics 74: 409-422.

25. Levin SR, Harley ET, Fackler JC, Lehmann CU, Custer JW, et al. (2012) Real-time forecasting of pediatric intensive care unit length of stay using computerized provider orders. Critical Care Medicine 40: 3058-3064.

26. Garcia AM (1991) System and method for scheduling and reporting patient related services including prioritizing services. Google Patents.

27. Systems PP (2015) Discharge planning, healthcare software, discharge referral system.

28. Calkins DR, Davis RB, Reiley P (1997) PAtient-physician communication at hospital discharge and patients\&\#39; understanding of the postdischarge treatment plan. Archives of Internal Medicine 157: 1026-1030.

29. Moran WP, Davis KS, Moran TJ, Newman R, Mauldin PD (2012) Where are my patients? It is time to automate notification of hospital use to primary care practices. Southern Medical Journal 105: 18-23.

30. Pethybridge $\mathrm{J}$ (2004) How team working influences discharge planning from hospital: A study of four multi-disciplinary teams in an acute hospital in England. Journal of Interprofessional Care 18: 29-41.

31. Anthony M, Hudson BD (1998) Successful patient discharge. A comprehensive model of facilitators and barriers. J Nurs Adm 28: 48-55.

32. Justinia T (2014) Implementing large-scale healthcare information systems. 